\title{
Một số nhân tố ảnh hưởng đến rủi ro tài chính - Nghiên cứu doanh nghiệp bất động sản niêm yết trên Sở Giao dịch Chứng khoán Thành phố Hồ Chí Minh (HSX)
}

\section{Some factors affect financial risk - A study from real estate companies listed on the Ho Chi Minh City Stock Exchange (HSX)}

\author{
Võ Minh Long ${ }^{1 *}$
}

${ }^{1}$ Trường Đại học Mở Thành phố Hồ Chí Minh, Việt Nam

"Tác giả liên hệ, Email: long.vm@ou.edu.vn

THÔNG TIN

DOI: $10.46223 / \mathrm{HCMCOUJS}$.

econ.vi.15.2.240.2020

Ngày nhận: 02/04/2020

Ngày nhận lại: 14/04/2020

Duyệt đăng: 22/04/2020

Tì khóa:

bất động sản, FEM - Ước lượng vững, HSX, rủi ro tài chính

Keywords:

real estate

FEM-Firm estimates

HSX, financial risk

\section{TÓM TÁT}

Bài viết xác định sự tác động của một số nhân tố đến rủi ro tài chính đối với các doanh nghiệp bất động sản niêm yết trên Sở Giao dịch chứng khoán Thành phố Hồ Chí Minh (HSX). Kết quả hồi quy FEM - ước lượng vững cho thấy rằng: Tỷ số thanh toán ngắn hạn, tỷ số sinh lời của tổng tài sản, vòng quay tồn kho, và tỷ số tự tài trợ có tác động cùng chiều với rủi ro tài chính các biến, như: Hệ số nợ ngắn hạn trên nợ dài hạn, tỷ số thanh toán nhanh, tỷ số thanh toán tổng quát, vòng quay tổng tài sản, và vòng quay khoản phải thu lại có kết quả ngược lại và tất cả đều có ý nghĩa thống kê cao. Ngoài ra, nó cũng cho biết thêm: Tỷ số sinh lời của doanh thu, vòng quay tài sản cố định, và tỷ số vốn cố định không có tác động đến rủi ro tài chính.

\section{ABSTRACT}

The paper identifies the impact of several factors on financial risk for real estate businesses listed on the Ho Chi Minh City Stock Exchange (HSX). The results of FEM-Firm estimates regression show that: Current ratio, return on assets, inventory turnover, and equity asset ratio have a positive effect on financial risk, but the factors such as short-term debt/longterm debt, quick ratio, asset liquid ratio, total assets turnover, and receivables turnover have the opposite result and all of them are statistically significant. In addition, the study also reveals that return on sales, fixed asset turnover, and fixed asset ratio have no impact on financial risk.

\section{Giới thiệu}

Bất động sản là ngành mang lại nhiều lợi nhuận nhưng cũng là một ngành nhạy cảm, nhiều rủi ro tiềm tàng. Mức độ rủi ro của nó phụ thuộc rất nhiều yếu tố, như: Chính sách của 
nhà nước, chính sách tài chính ngành và công ty...đặc biệt là sự biến động của giá cả trên thị trường bất động sản. Trước năm 2008, ngành bất động sản phát triển rất nhanh và mạnh, giá nhà đất liên tục tăng mạnh, điều này giúp doanh nghiệp ngành bất động sản hầu như đều có hiệu quả cao, tài sản có tính thanh khoản cao, đảm bảo trả nợ tốt cho ngân hàng... Tuy nhiên, từ năm 2008 (bắt đầu giai đoạn khủng hoảng) nền kinh tế Việt Nam nói riêng và thế giới nói chung cộng với các chính sách của Nhà nước để lành mạnh thị trường bất động sản, các doanh nghiệp trong ngành bất động sản gặp rất nhiều khó khăn: Không huy động được vốn, thua lỗ, mất khả năng thanh khoản, không trả được nợ ngân hàng... thậm chí là phá sản. Đó chính là rủi ro tài chính mà các doanh nghiệp bất động sản gặp phải trong quá trình hoạt động. Ngoài ra, theo lược khảo của tác giả về sự tác động một số nhân tố đến rủi ro tài chính, như: Bhunia và Mukhuti (2012), Gang, Weilan, và Liu (2012), Simantinee và Phani (2015) hay Vu (2017), ... lại cho các kết quả nghiên cứu tương đối khác nhau với sự không giống nhau về đối tượng, không gian, thời gian của các nhân tố tác động đến rủi ro tài chính. Với một số lý do trên, theo tác giả cần phải có nghiên cứu khoa học: Một số nhân tố ảnh hưởng đến rủi ro tài chính - nghiên cứu ngành bất động sản niêm yết trên Sở Giao dịch chứng khoán Thành phố Hồ Chí Minh (HSX) để giúp các nhà quản trị ngành bất động sản có cơ sở khoa học nhằm giảm thiểu rủi ro tài chính, góp phần giúp doanh nghiệp phát triển bền vững.

\section{Tổng hợp lý thuyết}

Theo Li (2003): Rủi ro tài chính là một khái niệm gắn liền với sự không chắc chắn của sự biến động các yếu tố, như: Lãi suất, tỷ giá, giá cổ phiếu, và giá cả hàng hóa. Tuy nhiên, Defan (2005) cho rằng: Rủi ro tài chính, bao gồm: Rủi ro theo nghĩa rộng và rủi ro theo nghĩa hẹp. Theo nghĩa rộng, rủi ro tài chính liên quan đến tất cả các yếu tố phản ánh trong tình hình tài chính doanh nghiệp. Theo nghĩa hẹp, rủi ro tài chính đề cập đến khả năng không thanh toán được các khoản nợ tài chính khi đến hạn. Ngoài ra, theo Zhe, Ke, Kaibi, và Xiaoliu (2012), rủi ro tài chính là xác suất mất vốn khi sử dụng các phương pháp tài trợ, điều này có thể làm giảm khả năng hoạt động trở lại của doanh nghiệp. Tóm lại, mặc dù hiện nay có nhiều quan điểm khác nhau về rủi ro tài chính nhưng bài viết này chỉ tập trung vào rủi ro tài chính, đó là những rủi ro phát sinh trong quá trình hoạt động của doanh nghiệp, làm ảnh hưởng đến tình hình tài chính, khả năng sinh lời của doanh nghiệp và nặng nề nhất là mất khả năng thanh toán các khoản nợ dẫn đến phá sản doanh nghiệp. Ngoài ra, khi doanh nghiệp bị rủi ro tài chính, thường họ sẽ bị tác động tiêu cực nhiều hơn tích cực, như: Tác động làm tăng chi phí của doanh nghiệp (cả chi phí tài chính và chi phí kinh doanh), vì vậy, nó ảnh hưởng đến lợi nhuận của doanh nghiệp. Ngoài ra, nó sẽ tác động đến khả năng chịu đựng tài chính và tái đầu tư của doanh nghiệp cũng như sự cân đối dòng tiền và khả năng thanh toán của doanh nghiệp và quan trọng nhất là, tác động đến giá trị doanh nghiệp và nặng nề hơn, sẽ làm cho các doanh nghiệp bị phá sản.

Với các nền tảng về rủi ro tài chính của các nhà nghiên cứu lý thuyết, họ được ủng hộ bởi các nhà nghiên cứu thực nghiệm về sự tác động của các nhân tố đến rủi ro tài chính, như: Kết quả nghiên cứu của Defang và cộng sự (2005) chỉ ra rằng: Quy mô và cơ cấu nợ có mối quan hệ cùng chiều với rủi ro tài chính nhưng hiệu suất hoạt động và khả năng sinh lời lại cho kết quả ngược lại. Ngoài ra, kết quả cũng cho biết: Chưa có cơ sở khoa học về mối quan hệ của lãi suất đi vay và khả năng thanh toán với rủi ro tài chính. Ngoài ra, kết quả nghiên cứu của 
Simantinee và Phani (2015): Vòng quay tài sản cố định, thu nhập mỗi cổ phiếu, tỷ suất lợi nhuận thuần, tỷ suất lợi nhuận ròng, hệ số khả năng thanh toán ngắn hạn và tỷ lệ thuế có tương quan cùng chiều với rủi ro tài chính, nhưng lại cho kết quả ngược lại cho các biến: Lợi nhuận thuần từ hoạt động trên mỗi cổ phiếu, lợi nhuận trên vốn dài hạn, hệ số khả năng thanh toán nhanh và hệ số khả năng thanh toán lãi vay. Đồng thời nó cũng cho biết: Không có mối tương quan của vòng quay hàng tồn kho và vòng quay tổng tài sản với rủi ro tài chính. Bên cạnh đó, kết quả nghiên cứu của $\mathrm{Vu}$ (2017) cho biết: Khả năng thanh toán, vòng quay hàng tồn kho, hệ số tự tài trợ và hệ số vốn cố định có tác động tiêu cực đến rủi ro tài chính, nhưng các chỉ tiêu: Hệ số nợ ngắn hạn trên nợ dài hạn, hệ số sinh lời của doanh thu, hệ số sinh lời của tổng tài sản, vòng quay tài sản cố định, vòng quay tổng tài sản và vòng quay các khoản phải thu không ảnh hưởng đến rủi ro tài chính.

Như vậy, cả lý thuyết cũng như nghiên cứu thực nghiệm đều cho rằng: Rủi ro tài chính chịu tác động của nhiều nhân tố và nó ảnh hưởng đến tình hình hoạt động cũng như giá trị doanh doanh nghiệp. Đây chính là cơ sở quan trọng để bài viết đề xuất mô hình nghiên cứu về một số nhân tố ảnh hưởng đến rủi ro tài chính - nghiên cứu doanh nghiệp bất động sản niêm yết trên sở giao dịch chứng khoán Thành Phố Hồ Chí Minh (HSX) nhằm bổ sung thêm bằng chứng khoa học về các nhân tố tác động đến rủi ro tài chính cũng như giúp nhà quản lý có cơ sở khoa học để đưa giải pháp nhằm giảm thiểu rủi ro tài chính giúp họ nâng cao hiệu quả, giá trị doanh nghiệp và ổn định để phát triển.

\section{Phương pháp nghiên cứu}

\subsection{Mô hình nghiên cúu}

Căn cứ vào lý thuyết và kế thừa từ một số tác giả nghiên cứu về rủi ro tài chính, như: Bathory (1984), Gang và cộng sự (2012) và Vu (2017), có 5 yếu tố chính ảnh hưởng đến rủi ro tài chính của các công ty niêm yết ngành bất động sản niêm yết trên HSX, bao gồm: Cơ cấu nợ (DS), khả năng thanh toán $(\mathrm{SO})$, khả năng sinh lời $(\mathrm{PR})$, hiệu suất hoạt động $(\mathrm{PE})$ và cơ cấu vốn $(\mathrm{CS})$. Theo đó, 12 tỷ số tài chính được xem như là các biến độc lập để diễn tả 5 yếu tố chính ảnh hưởng đến rủi ro tài chính của 28 doanh nghiệp bất động sản niêm yết trên HSX giai đoạn 2012-2018 và nó được trình bày trong Bảng 1 . Ngoài ra, lý thuyết cũng như các nghiên cứu thực nghiệm của các tác giả trên cũng cho rằng: Giá trị của mô hình $(\mathrm{FR})$ càng nhỏ $=>$ Rủi ro tài chính của doanh nghiệp càng cao và ngược lại.

Mô hình tổng quát một số nhân tố ảnh hưởng đến rủi ro tài chính với biến phụ thuộc: Rủi ro tài chính (FR) và 12 tỷ số tài chính: Hệ số nợ ngắn hạn trên nợ dài hạn, tỷ số thanh toán ngắn hạn, tỷ số thanh toán nhanh, tỷ số thanh toán tổng quát, tỷ số sinh lời của doanh thu, tỷ số sinh lời của tổng tài sản, vòng quay tồn kho, vòng quay tài sản cố định, vòng quay tổng tài sản, vòng quay khoản phải thu, tỷ số tự tài trợ, và tỷ số vốn cố định như sau:

$$
\begin{aligned}
& \mathrm{FR}_{i t}=\beta_{0}+\beta_{1} \mathrm{SLD}_{\mathrm{it}}+\beta_{2} \mathrm{CR}_{\mathrm{it}}+\beta_{3} \mathrm{QR}_{\mathrm{it}}+\beta_{4} \mathrm{ALR}_{\mathrm{it}}+\beta_{5} \mathrm{ROS}_{\mathrm{it}}+\beta_{6} \mathrm{ROA}_{\mathrm{it}}+\beta_{7 \mathrm{IT}} \mathrm{it} \\
& \quad+\beta_{8} \mathrm{FAT}_{\mathrm{it}}+\mathrm{B}_{9} \mathrm{TAT}_{\mathrm{it}}+\beta_{10 \mathrm{RT}}+\beta_{11} \mathrm{EAR}_{\mathrm{it}}+\beta_{12} \mathrm{FAR}_{\mathrm{it}}+\mathrm{u}_{\mathrm{it}}
\end{aligned}
$$




\section{Bảng 1}

Định nghĩa và mô tả các biến

\begin{tabular}{|c|c|c|c|}
\hline Tên biến & $\begin{array}{c}\text { Ký hiệu } \\
\text { biến }\end{array}$ & Định nghĩa & Mô tả \\
\hline Rủi ro tài chính & FR & Rủi ro tài chính & $\begin{array}{l}\text { (Lợi nhuận trước thuế + Khấu hao } \\
\text { tài sản cố định + Thuế thu nhập } \\
\text { hoãn lại)/Nợ ngắn hạn }\end{array}$ \\
\hline Cơ cấu nợ (DS) & SLD & $\begin{array}{l}\text { Hệ số nợ ngắn hạn trên } \\
\text { nợ dài hạn }\end{array}$ & Nợ ngắn hạn/Nợ dài hạn \\
\hline \multirow{3}{*}{$\begin{array}{l}\text { Khả năng thanh } \\
\text { toán (SO) }\end{array}$} & $\mathrm{CR}$ & $\begin{array}{l}\text { Tỷ số thanh toán ngắn } \\
\text { hạn }\end{array}$ & Tài sản ngắn hạn/Nợ ngắn hạn \\
\hline & QR & Tỷ số thanh toán nhanh & $\begin{array}{l}\text { (Tài sản ngắn hạn - tồn kho)/Nợ } \\
\text { ngắn hạn }\end{array}$ \\
\hline & ALR & $\begin{array}{l}\text { Tỷ số thanh toán tổng } \\
\text { quát }\end{array}$ & Tổng tài sản/Tổng nợ phải trả \\
\hline \multirow{2}{*}{$\begin{array}{l}\text { Khả năng sinh lời } \\
\text { (PR) }\end{array}$} & ROS & $\begin{array}{l}\text { Tỷ số sinh lời của doanh } \\
\text { thu }\end{array}$ & $\begin{array}{l}\text { Lợi nhuận sau thuế/Doanh thu } \\
\text { thuần }\end{array}$ \\
\hline & ROA & $\begin{array}{l}\text { Tỷ số sinh lời của tổng } \\
\text { tài sản }\end{array}$ & $\begin{array}{l}\text { Lợi nhuận sau thuế/Tổng tài sản } \\
\text { bình quân }\end{array}$ \\
\hline \multirow{4}{*}{$\begin{array}{l}\text { Hiệu suất hoạt } \\
\text { dộng (PE) }\end{array}$} & IT & Vòng quay tồn kho & $\begin{array}{c}\text { Giá vốn hàng bán/Tồn kho bình } \\
\text { quân }\end{array}$ \\
\hline & FAT & $\begin{array}{l}\text { Vòng quay tài sản cố } \\
\text { định }\end{array}$ & $\begin{array}{l}\text { Doanh thu thuần/Tài sản cố định } \\
\text { bình quân }\end{array}$ \\
\hline & TAT & Vòng quay tổng tài sản & $\begin{array}{l}\text { Doanh thu thuần/Tổng tài sản bình } \\
\text { quân }\end{array}$ \\
\hline & RT & $\begin{array}{l}\text { Vòng quay khoản phải } \\
\text { thu }\end{array}$ & $\begin{array}{l}\text { Doanh thu thuần/Khoản phải thu } \\
\text { bình quân }\end{array}$ \\
\hline \multirow{2}{*}{ Cơ cấu vốn (CS) } & EAR & Tỷ số tự tài trợ & Vốn chủ sở hữu/Tổng tài sản \\
\hline & FAR & Tỷ số vốn cố định & Tài sản cố định/Tổng tài sản \\
\hline
\end{tabular}

Nguồn: Tác giả tổng hợp 


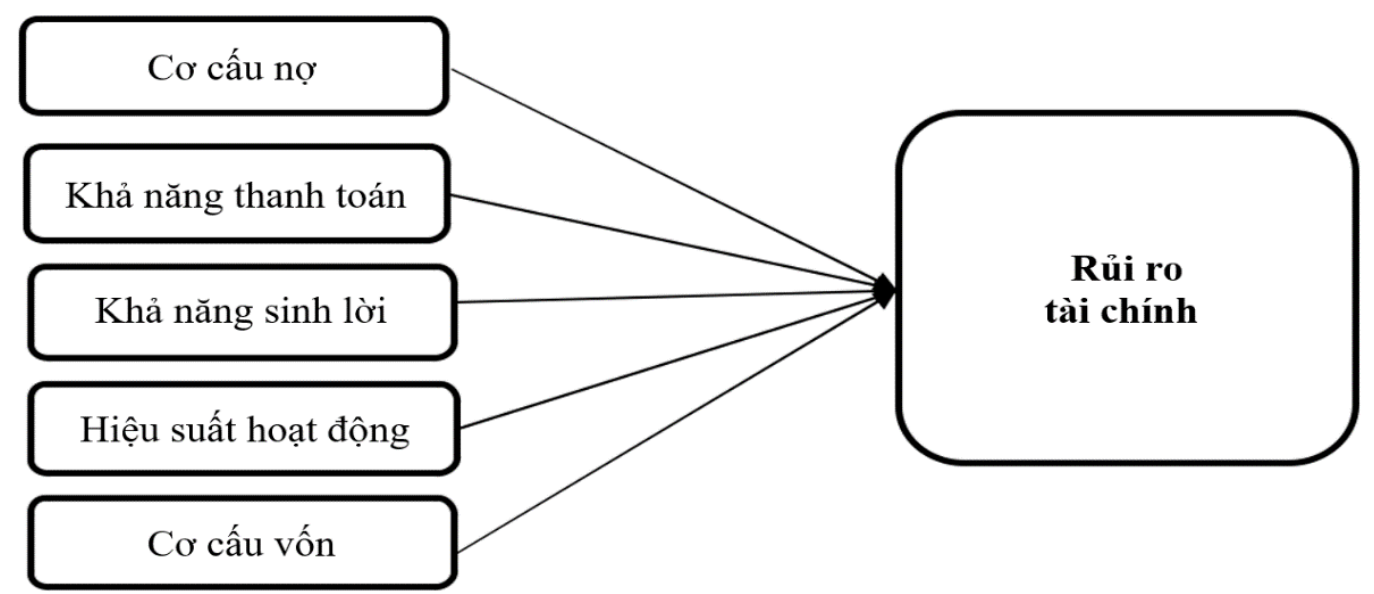

Nguồn: Đề xuất của tác giả

Hình 1. Sơ đồ mô hình nghiên cứu

\subsection{Dũ liệu nghiên cứu và phương pháp nghiên cúu}

Dữ liệu nghiên cứu được thu thập từ các báo cáo tài chính thường niên đã được kiểm toán của 28 doanh nghiệp trong ngành bất động sản đang hoạt động trong giai đoạn từ năm 2012 đến năm 2018. Như vậy, bài nghiên cứu có tổng cộng 196 biến quan sát (28 doanh nghiệp * 7 năm), đã qua tổng hợp, xử lý và thiết kế bằng phần mềm Excel. Như vậy, với số mẫu nghiên cứu này được xem đủ lớn và có ý nghĩa thống kê. Với dữ liệu này, bài viết sử dụng phần mềm Stata 14.0 để thực hiện hồi quy và các kiểm định cần thiết. Để có kết quả nghiên cứu, bài viết sử dụng một số phương pháp hồi quy, như: Pooled OLS, FEM, REM và FEM ước lượng vững. Ngoài ra, sau khi thực hiện hồi quy với các phương pháp trên, bài viết sẽ thực hiện các kiểm định cần thiết để so sánh các phương pháp hồi quy cũng như các vi phạm trong mô hình nhằm lựa chọn phương pháp phù hợp nhất với mục tiêu nghiên cứu, như: kiểm định $\mathrm{F}$, LM, Hausman, hiện tượng đa cộng tuyến, phương sai thay đổi cũng như hiện tượng tự tương quan. Sau cùng, khi đã có kết quả nghiên cứu, bài viết sử dụng các lý thuyết đã nghiên cứu kết hợp với thực tế khách quan để lý giải về ý nghĩa kinh tế của mối tương quan giữa các biến giải thích và biến độc lập để làm rõ kết quả nghiên cứu.

\section{Kết quả nghiên cứu}

\subsection{Kiểm tra tính dùng}

\section{Bảng 2}

Kết quả kiểm tra tính dừng của các biến tại bậc gốc

\begin{tabular}{|c|c|c|c|}
\hline \multirow{2}{*}{ Biến } & \multicolumn{2}{|c|}{ Kiểm định Levin-Lin-Chu } & \multirow{2}{*}{ Kết quả } \\
\cline { 2 - 3 } & t-Statistic & P-value & \\
\hline FR & -45.6044 & 0.0000 & Dừng \\
\hline SLD & $-2.4 \mathrm{e}+03$ & 0.0000 & Dừng \\
\hline CR & -31.3201 & 0.0000 & Dừng \\
\hline QR & -48.6685 & 0.0000 & Dừng \\
\hline ALR & -13.2908 & 0.0000 & Dừng \\
\hline
\end{tabular}




\begin{tabular}{|c|c|c|c|}
\hline \multirow{2}{*}{ Biến } & \multicolumn{2}{|c|}{ Kiểm định Levin-Lin-Chu } & \multirow{2}{*}{ Kết quả } \\
\cline { 2 - 4 } & t-Statistic & P-value & Dừng \\
\hline ROS & -4.7266 & 0.0000 & Dừng \\
\hline ROA & -11.1867 & 0.0000 & Dừng \\
\hline IT & -26.4730 & 0.0000 & Dừng \\
\hline FAT & -27.5989 & 0.0000 & Dừng \\
\hline TAT & -23.7484 & 0.0000 & Dừng \\
\hline RT & -15.6474 & 0.0000 & Dừng \\
\hline EAR & -11.6555 & 0.0000 & Dừng \\
\hline FAR & -15.9710 & 0.0000 & \\
\hline
\end{tabular}

Nguồn: Trích xuất từ phần mềm Stata 14.0

Kết quả kiểm định theo Levin, Lin, và Chu (2002), cho thấy: Chuỗi dữ liệu gốc dừng ở mức ý nghĩa với $\mathrm{P}$-value $=0.0000<\alpha=1 \%$. Do đó, các biến nghiên cứu đều có ý nghĩa thống kê.

\subsection{Phân tích thống kê mô tả}

\section{Bảng 3}

Kết quả thống kê mô tả

\begin{tabular}{|c|c|c|c|c|c|}
\hline Variables & Obs & Mean & Std. Dev. & Min & Max \\
\hline FR & 196 & 1.8646 & 2.1848 & $(0.3285)$ & 18.4273 \\
\hline SLD & 196 & 16.2355 & 70.9394 & 0.0887 & 702.0591 \\
\hline CR & 196 & 3.3301 & 4.7775 & 0.4213 & 42.7145 \\
\hline QR & 196 & 1.3987 & 1.7752 & 0.0723 & 12.3161 \\
\hline ALR & 196 & 2.7073 & 3.2444 & 1.0924 & 32.3389 \\
\hline ROS & 196 & 0.1398 & 0.5463 & $(5.7505)$ & 1.2145 \\
\hline ROA & 196 & 0.0456 & 0.0566 & $(0.1628)$ & 0.3208 \\
\hline IT & 196 & 1.0689 & 1.7999 & 0.0352 & 12.7289 \\
\hline FAT & 196 & 2.0457 & 1.1196 & 0.6557 & 5.7382 \\
\hline TAT & 196 & 0.3416 & 0.5307 & 0.0095 & 4.2626 \\
\hline RT & 196 & 0.4004 & 1.0974 & 2.6619 & 3.0380 \\
\hline EAR & 196 & 0.4802 & 0.1756 & 0.0846 & 0.9691 \\
\hline FAR & 196 & 0.0456 & 0.0485 & 0.0014 & 0.2402 \\
\hline
\end{tabular}

Nguồn: Kết quả phân tích từ phần mềm STATA 14.0 
Bảng thống kê mô tả, chỉ ra rằng: Giá trị của biến rủi ro tài chính $(\mathrm{FR})$ trong giai đoạn nghiên cứu có giá trị nhỏ nhất là -0.3285 , giá trị lớn nhất là 18.4273 , giá trị trung bình là 1.8646 và độ lệch chuẩn là 2.1848 . Điều này có nghĩa các giá trị của rủi ro tài chính trong mẫu nghiên cứu biến thiên khá lớn từ -0.3285 đến 18.4273 . Tương tự như biến rủi ro tài chính, đa phần các biến còn lại đều có sự biến thiên khá lớn, ngoại trừ biến ROA và RT.

\subsection{Phân tích hệ số tương quan}

\section{Bảng 4}

Kết quả hệ số tương quan Pearson (r)

\begin{tabular}{|c|c|c|c|c|c|c|c|c|c|c|c|c|}
\hline & SLD & CR & QR & ALR & ROS & ROA & IT & FAT & TAT & RT & EAR & FAR \\
\hline SLD & 1.0000 & & & & & & & & & & & \\
\hline CR & 0.0431 & 1.0000 & & & & & & & & & & \\
\hline QR & 0.1625 & 0.8249 & 1.0000 & & & & & & & & & \\
\hline ALR & 0.4556 & 0.6421 & 0.6965 & 1.0000 & & & & & & & & \\
\hline ROS & 0.0105 & 0.0943 & 0.1592 & 0.0748 & 1.0000 & & & & & & & \\
\hline ROA & $(0.0380)$ & $0.0035)$ & 0.0971 & $(0.0184)$ & 0.5138 & 1.0000 & & & & & & \\
\hline IT & 0.0768 & $(0.1275)$ & 0.0119 & $(0.0057)$ & 0.0080 & 0.1418 & 1.0000 & & & & & \\
\hline FAT & $(0.0136)$ & $(0.0089)$ & $(0.0794)$ & $(0.0067)$ & 0.0833 & 0.1677 & 0.1561 & 1.0000 & & & & \\
\hline TAT & 0.0177 & $(0.1185)$ & $(0.0677)$ & $(0.0532)$ & $(0.0074)$ & 0.1699 & 0.8886 & 0.2685 & 1.0000 & & & \\
\hline RT & 0.0707 & 0.0378 & 0.1441 & 0.0832 & 0.1709 & 0.4399 & 0.4097 & 0.2068 & 0.4864 & 1.0000 & & \\
\hline EAR & 0.3204 & 0.3566 & 0.3934 & 0.5678 & 0.1808 & 0.1773 & $(0.0852)$ & $(0.0606)$ & $(0.0999)$ & 0.1017 & 1.0000 & \\
\hline FAR & $(0.0007)$ & $(0.0584)$ & 0.0968 & $(0.0339)$ & 0.0899 & 0.1352 & 0.5537 & $(0.4958)$ & 0.5003 & 0.4052 & 0.0002 & 1.0000 \\
\hline
\end{tabular}

Nguồn: Kết quả phân tích từ phần mềm STATA 14.0

Với kết quả ở Bảng 4, cho thấy: Giữa các cặp biến độc lập có mối tương quan rất thấp, ngoại trừ các cặp biến: $C R$ và $Q R ; C R$ và $A L R ; Q R$ và $A L R ; I T$ và $T A T$. Điều này có thể nhận định rằng: Các biến trong mô hình có thể có hiện tượng đa cộng tuyến với nhau. Vì vậy, bài viết sẽ tiếp tục kiểm tra hiện tượng đa cộng tuyến để khắc phục các khuyết tật của mô hình (nếu có).

\subsection{Kiểm định hiện tuọng đa cộng tuyến}

\section{Bảng 5}

Kết quả kiểm định hiện tượng đa cộng tuyến

\begin{tabular}{|c|c|c|c|c|c|c|c|c|c|c|c|c|}
\hline Variable & SLD & CR & QR & ALR & ROS & ROA & IT & FAT & TAT & RT & EAR & FAR \\
\hline VIF & 1.49 & 4.03 & 4.55 & 3.29 & 1.46 & 1.76 & 5.96 & 3.18 & 6.71 & 2 & 1.63 & 4.4 \\
\hline 1/VIF & 0.6716 & 0.2483 & 0.2196 & 0.3038 & 0.6845 & 0.5686 & 0.1678 & 0.3148 & 0.1490 & 0.5008 & 0.6139 & 0.2274 \\
\hline
\end{tabular}

Nguồn: Kết quả phân tích từ phần mềm STATA 14.0

Theo Hoàng Trọng và cộng sự (2008), kết quả có giá trị VIF < 7. Kết luận: Các biến trong mô hình không có hiện tượng đa cộng tuyên. 


\subsection{Kiểm tra lựa chọn mô hình}

\section{Bảng 6}

Kết quả kiểm định lựa chọn mô hình

\begin{tabular}{|l|l|c|}
\hline \multicolumn{1}{|c|}{ Kiểm định } & \multicolumn{1}{c|}{ Thống kê } & Kết quả \\
\hline F-test (Pooled OLS và FEM) & Prob>F $=0.0000$ & FEM \\
\hline Hausman test (FEM và REM) & Prob>chi $2=0.0016$ & FEM \\
\hline
\end{tabular}

Nguồn: Trích xuất từ phần mềm Stata 14.0

Kết luận: Căn cứ kết quả kiểm định ở Bảng 6: Phương pháp FEM được lựa chọn.

\subsection{Kiểm định hiện tự̣ng phuơng sai thay đổi và tụ tương quan}

\section{Bảng 7}

Kết quả kiểm định hiện tượng phương sai thay đổi và tự tương quan

\begin{tabular}{|c|c|c|}
\hline \multicolumn{1}{|c|}{ Kiểm định } & \multicolumn{1}{|c|}{ Thống kê } & \multicolumn{1}{c|}{ Kết quả } \\
\hline Phương sai thay đổi (Wald) & Prob $>$ chi2 $=0.0000<\alpha=1 \%$ & $\begin{array}{l}\text { Có hiện tượng phương } \\
\text { sai thay đồi }\end{array}$ \\
\hline Tự tương quan (Wooldrige) & Prob $>\mathrm{F}=0.2162>\alpha=5 \%$ & $\begin{array}{l}\text { Không có hiện tượng tự } \\
\text { tương quan }\end{array}$ \\
\hline
\end{tabular}

Nguồn: Kết quả phân tích từ phần mềm STATA 14.0

\subsection{Kết quả phân tích hồi quy}

Sau khi thực hiện các kiểm định, kết quả cho thấy: Tất cả các chuỗi số liệu của từng biến đều dừng và có ý nghĩa thống kê. Ngoài ra, nó cũng cho biết: Có hiện tượng phương sai thay đổi nên bài viết sử dụng ước lượng mô hình tác động cố định vững (FEM ước lượng vững) để khắc phục khuyết tật này, do đó, kết quả và thảo luận kết quả nghiên cứu được thực hiện theo ước lượng này.

\section{Bảng 8}

Tổng hợp kết quả hồi quy của các mô hình Pooled OLS, FEM, REM và FEM ước lượng vững

\begin{tabular}{|c|c|c|c|c|}
\hline Biến & Pooled OLS & FEM & REM & FEM ước lượng vũng \\
\hline SLD & -0.00046 & -0.000757 & -0.000631 & $-0.000757 * * *$ \\
\hline & $(-0.69)$ & $(-1.50)$ & $(-1.28)$ & $(-2.98)$ \\
\hline CR & $0.488 * * *$ & $0.519 * * *$ & $0.512 * * *$ & $0.519 * * *$ \\
\hline & $(-30.2)$ & $(-37.34)$ & $(-38.52)$ & $(-62.06)$ \\
\hline QR & $-0.263 * * *$ & $-0.329 * * *$ & $-0.313 * * *$ & $-0.329 * * *$ \\
\hline & $(-5.68)$ & $(-8.33)$ & $(-8.54)$ & $(-14.95)$ \\
\hline ALR & $-0.0589 * * *$ & $-0.0789 * *$ & $-0.0765 * * *$ & $-0.0789 * * *$ \\
\hline & $(-2.74)$ & $(-4.78)$ & $(-4.79)$ & $(-6.65)$ \\
\hline ROS & 0.079 & -0.0126 & 0.00309 & -0.0126 \\
\hline
\end{tabular}




\begin{tabular}{|c|c|c|c|c|}
\hline Biến & Pooled OLS & FEM & REM & FEM ước lượng vũng \\
\hline & $(-0.93)$ & $(-0.20)$ & $(-0.05)$ & $(-0.62)$ \\
\hline ROA & $6.392^{* * *}$ & $5.846^{* * *}$ & $5.913 * * *$ & $5.846^{* * *}$ \\
\hline & $(-7.1)$ & $(-8.8)$ & $(-9)$ & $(-11.84)$ \\
\hline IT & -0.0209 & 0.0377 & 0.0262 & $0.0377 * * *$ \\
\hline & $(-0.40)$ & $(-0.88)$ & $(-0.63)$ & $(-3.61)$ \\
\hline FAT & $-0.106 *$ & 0.0674 & 0.0282 & 0.0674 \\
\hline TAT & $(-1.73)$ & $(-1.13)$ & $(-0.51)$ & $(-1.41)$ \\
\hline & 0.122 & $-0.352^{*}$ & -0.259 & $-0.352^{* * *}$ \\
\hline RT & $(-0.65)$ & $(-1.74)$ & $(-1.43)$ & $(-9.54)$ \\
\hline & $-0.0995 * *$ & $-0.102^{*}$ & $-0.0890 *$ & $-0.102^{* *}$ \\
\hline EAR & $(-2.01)$ & $(-1.74)$ & $(-1.75)$ & $(-2.25)$ \\
\hline & $3.001 * * *$ & $2.937 * *$ & $3.004 * * *$ & $2.937 * * *$ \\
\hline FAR & $(-10.74)$ & $(-9.11)$ & $(-10.49)$ & $(-12.9)$ \\
\hline & $-3.377 * *$ & -0.634 & -0.83 & -0.634 \\
\hline cons & $(-2.03)$ & $(-0.40)$ & $(-0.56)$ & $(-1.44)$ \\
\hline & $-0.578 * * *$ & $-0.841 * * *$ & $-0.821 * * *$ & $-0.841^{* * *}$ \\
\hline R - Squared & $(-2.85)$ & $(-4.03)$ & $(-3.95)$ & $(-7.19)$ \\
\hline & 0.9433 & 0.9584 & 0.9582 & 0.9584 \\
\hline
\end{tabular}

Ghi chú: *,**,*** tương ứng mức ý nghĩa $10 \%, 5 \%, 1 \%$. Giá trị thống kê $\mathrm{t}$ trong ()

Nguồn: Kết quả phân tích từ phần mềm STATA 14.0

Để làm rõ kết quả nghiên cứu, tác giả đi vào phần thảo luận kết quả.

\subsection{Thảo luận kết quả nghiên cứu}

4.8.1. Nhóm biến có tác động cùng chiều đến rủi ro tài chính

\section{Bảng 9}

Nhóm biến có tác động cùng chiều đến rủi ro tài chính

\begin{tabular}{|c|c|c|c|c|}
\hline Biến & Pooled OLS & FEM & REM & FEM ước lượng vũng \\
\hline CR & $0.488^{* * *}$ & $0.519 * * *$ & $0.512 * * *$ & $0.519 * * *$ \\
\hline ROA & $6.392 * * *$ & $5.846 * * *$ & $5.913 * * *$ & $5.846 * * *$ \\
\hline IT & -0.0209 & 0.0377 & 0.0262 & $0.0377 * * *$ \\
\hline EAR & $3.001 * * *$ & $2.937 * * *$ & $3.004 * * *$ & $2.937 * * *$ \\
\hline
\end{tabular}

Ghi chú: *, **, *** tương ứng mức ý nghĩa 10\%, 5\%, 1\%.

Nguồn: Tổng hợp của tác giả.

- Đối với tỷ số thanh toán ngắn hạn: Biến này có tác động tích cực $(+)$ đến biến FR, tức là, khi tỷ số thanh toán ngắn hạn tăng lên sẽ làm cho rủi ro tài chính giảm xuống và nó có độ tin cậy $99 \%$. Điều này có thể giải thích: Tỷ số thanh toán ngắn hạn tăng lên tức là doanh nghiệp có thể đảm bảo được khả năng thanh toán các khoản nợ ngắn hạn và góp phần giảm rủi ro tài chính. Kết quả nghiên cứu này cũng phù hợp với kỳ vọng của tác giả và nó được ủng hộ của một số tác giả, như: Gang và cộng sự (2012), Bhunia và Mukhuti (2012), Simantinee và Phani (2015). 
- Đối với tỷ số sinh lời của tổng tài sản: Nó có tác động tích cực (+) đến biến FR, nghĩa là, khi tỷ số sinh lời của tổng tài sản tăng lên sẽ làm cho rủi ro tài chính giảm xuống và nó có mức ý nghĩa thống kê $1 \%$. Theo tác giả, khi doanh nghiệp cải thiện hiệu quả sinh lời sẽ giúp họ tăng vốn chủ sở hữu cũng như tăng khả năng thanh toán các khoản nợ, thúc đẩy doanh nghiệp đầu tư lớn hơn để mở rộng quy mô, phát triển và chiếm giữ thị phần và nó sẽ làm giảm rủi ro tài chính. Kết quả nghiên cứu này được sự ủng hộ của một số tác giả, như: Gang và cộng sự (2012), Bhunia và Mukhuti (2012).

- Đối với vòng quay hàng tồn kho: Biến này có tác động tích cực $(+)$ đối với $\mathrm{FR}$, tức là, khi doanh nghiệp tăng vòng quay hàng tồn kho sẽ giúp giảm rủi ro tài chính và có độ tin cậy $99 \%$. Theo tác giả, Nếu doanh nghiệp có vòng quay hàng tồn kho cao, tức họ đã đẩy mạnh việc tiêu thụ hàng hóa, góp phần giải quyết hàng tồn kho rất tốt - Nếu hàng hóa tồn đọng quá nhiều và kéo dài sẽ làm cho doanh nghiệp tăng các khoản chi phí, như: Lưu kho, bảo quản kho.... kể cả chi phí cơ hội do đầu tư vào khoản tồn kho cũng như họ không bán được hàng và là nguyên nhân không tạo được dòng tiền để trả nợ => Làm tăng rủi ro. Kết quả nghiên cứu ngược chiều với kết quả nghiên cứu của Simantinee và Phani (2015).

- Đối với tỷ số tự tài trợ: Nó có tác động cùng chiều (+) đối với biến FR, nghĩa là, khi doanh nghiệp tăng hệ số tự tài trợ sẽ giúp giảm rủi ro tài chính và có độ tin cậy $99 \%$. Theo bài viết, nếu doanh nghiệp tăng tỷ số này, tức là họ tăng vốn chủ sở hữu, điều này giúp doanh nghiệp chủ động trong việc sử dụng nguồn vốn cũng như giúp họ dễ dàng thanh toán các khoản nợ, góp phần cải thiện rủi ro tài chính. Kết quả nghiên cứu phù hợp với kỳ vọng của tác giả và nó cũng được sự ủng hộ của Gang và cộng sự (2012), Bhunia và Mukhuti (2012), Vu (2017). Hàm ý chính sách: Doanh nghiệp nên tăng vốn chủ sở hữu để giảm rủi ro tài chính, điều này giúp họ an tâm cho các hoạt động sản xuất, kinh doanh, chính nó góp phần cải thiện hiệu quả và nó cũng tác động tiếp tục làm giảm rủi ro tài chính.

\subsubsection{Nhóm biến có tác động trái chiều đến rủi ro tài chính}

\section{Bảng 10}

Nhóm biến có tác động trái chiều đến rủi ro tài chính

\begin{tabular}{|c|c|c|c|c|}
\hline Biến & Pooled OLS & FEM & REM & $\begin{array}{c}\text { FEM ước lượng } \\
\text { vũng }\end{array}$ \\
\hline SLD & -0.00046 & -0.000757 & -0.000631 & $-0.000757 * * *$ \\
\hline QR & $-0.263 * * *$ & $-0.329 * * *$ & $-0.313 * * *$ & $-0.329 * * *$ \\
\hline ALR & $-0.0589 * * *$ & $-0.0789 * * *$ & $-0.0765 * * *$ & $-0.0789 * * *$ \\
\hline TAT & 0.122 & $-0.352 *$ & -0.259 & $-0.352 * * *$ \\
\hline RT & $-0.0995 * *$ & $-0.102 *$ & $-0.0890 *$ & $-0.102 * *$ \\
\hline
\end{tabular}

Nguồn: Tổng hợp của tác giả

- Đối với hệ số nơ ngắn hạn trên nợ dài hạn: Kết quả nghiên cứu chỉ ra rằng: Biến này có tác động ngược chiều (-) với FR, tức là, khi doanh nghiệp tăng hệ số nợ ngắn hạn trên nợ dài hạn sẽ làm cho rủi ro tài chính tăng lên và có độ tin cậy $99 \%$. Theo tác giả, khi doanh nghiệp có hệ số này càng cao, tức là họ sẽ duy trì một mức nợ ngắn hạn cao nên khi dòng tiền của doanh nghiệp không đủ trả nợ sẽ làm cho doanh nghiệp mất khả năng thanh toán, không có khả 
năng trả nợ và điều này làm tăng rủi ro. Kết quả nghiên cứu này cũng phù hợp với kỳ vọng của tác giả. Hàm ý chính sách: Doanh nghiệp nên cơ cấu nợ theo hướng tăng nợ dài hạn và giảm nợ ngắn hạn nhằm giảm áp lực trả nợ cũng như tận dụng được đòn bẩy tài chính, góp phần cải thiện khả năng sinh lời từ đó giảm rủi ro tài chính.

- Đối với tỷ số thanh toán nhanh: Kết quả nghiên cứu cho biết: Hệ số này có tác động ngược chiều (-) với FR, tức là, khi doanh nghiệp tăng tỷ số thanh toán nhanh sẽ làm cho rủi ro tài chính tăng lên và có ý nghĩa thống kê ở mức $1 \%$. Theo tác giả, nếu doanh nghiệp tăng hệ số khả năng thanh toán nhanh, thường đồng nghĩa với việc giảm bớt lượng hàng tồn kho, tuy nhiên, trong trường hợp hàng tồn kho không đủ để đáp ứng nhu cầu thị trường, doanh nghiệp sẽ bị giảm hiệu quả hoạt động và nó là một trong những nguyên nhân làm tăng rủi ro tài chính doanh nghiệp. Kết quả nghiên cứu này được sự ủng hộ của Simantinee và Phani (2015).

- Đối với tỷ số khả năng thanh toán tổng quát: Tỷ số này có tác động ngược chiều (-) với FR, tức là, khi doanh nghiệp tăng tỷ số khả năng thanh toán tổng quát sẽ làm cho rủi ro tài chính tăng lên và có ý nghĩa thống kê ở mức $1 \%$. Theo bài viết, khi doanh nghiệp có tỷ số này càng cao, doanh nghiệp có tổng tài sản càng lớn và doanh nghiệp có tài sản lớn chưa hẳn là mang lại nhiều rủi ro nhưng với các doanh nghiệp bất động sản có quá nhiều hàng tồn kho chưa được thanh lý, những dự án dở dang chưa hoàn thành hoặc không đủ vốn để hoàn thành sẽ mang lại trở ngại cũng như làm tăng rủi ro cho doanh nghiệp. Kết quả nghiên cứu này trái ngược với kết quả nghiên cứu của Simantinee và Phani (2015), Vu (2017).

- Đối với vòng quay tổng tài sản: Biến này có tác động ngược chiều (-) với FR, tức là, khi doanh nghiệp tăng vòng quay tổng tài sản sẽ làm cho rủi ro tài chính tăng lên và có ý nghĩa thống kê ở mức $1 \%$ và nó cũng được ủng hộ bởi Gang và cộng sự (2012). Theo tác giả, những năm gần đây ngành bất động sản gặp không ít khó khăn, vì vậy, kết quả hoạt động kinh doanh của họ cũng bị giảm sút nên sự gia tăng vòng quay tổng tài sản làm cho hiệu quả hoạt động kém hiệu quả hơn và nó là nguyên nhân làm cho rủi ro tài chính tăng lên.

- Đối với vòng quay các khoản phải thu: Kết quả này cho biết: Vòng quay các khoản phải thu có tác động tiêu cực (-) với FR, tức là: Nếu doanh nghiệp tăng vòng quay các khoản phải thu thì rủi ro tài chính tăng. Kết quả nghiên cứu này phù hợp với kỳ vọng của tác giả. Theo tác giả, doanh nghiệp có vòng quay các khoản phải thu cao tức là các khoản phải thu càng nhỏ nhưng với các doanh nghiệp trong ngành bất động sản đều kinh doanh theo hình thức công nợ, việc các khoản phải thu giảm đồng nghĩa với doanh nghiệp không tiêu thụ được nhiều sản phẩm và nó góp phần làm giảm hiệu quả hoạt động và làm tăng rủi ro tài chính.

\subsubsection{Nhóm biến không có tác động đến rủi ro tài chính}

Kết quả nghiên cứu chỉ ra rằng: Chưa đủ cơ sở khoa học về sự tác động của các biến, như: ROS, FAT, FAR đến rủi ro tài chính.

\section{Kết luận, hạn chế và đề nghị}

\subsection{Kết luận}

Bài viết đã nêu bật được các cơ sở lý thuyết cũng như các nghiên cứu thực nghiệm về một số nhân tố tác động đến rủi ro tài chính và trên cơ sở đó, tác giả đã xây dựng mô hình nghiên cứu. Sau khi thực hiện các hồi quy cũng như kiểm định cần thiết để lựa chọn phương pháp phù hợp, kết quả nghiên cứu đã chỉ ra rằng: Chỉ có 4 biến, như: Tỷ số thanh toán ngắn 
hạn, tỷ số sinh lời của tổng tài sản, vòng quay tồn kho, và tỷ số tự tài trợ có tác động cùng chiều với rủi ro tài chính, tức là khi các tỷ số này tăng lên sẽ làm rủi ro tài chính giảm xuống, nhưng có đến 5 biến, như: Hệ số nợ ngắn hạn trên nợ dài hạn, tỷ số thanh toán nhanh, tỷ số thanh toán tổng quát, vòng quay tổng tài sản và vòng quay khoản phải thu lại có kết quả ngược lại, tức là khi các tỷ số này tăng lên sẽ làm rủi ro tài chính tăng lên. Tuy nhiên, nó cũng chỉ ra rằng: Không có mối quan hệ giữa 3 biến: Tỷ số sinh lời của doanh thu, vòng quay tài sản cố định, và tỷ số vốn cố định với rủi ro tài chính. Với kết quả nghiên cứu này, đây là cơ sở khoa học cho các nhà quản lý trong ngành bất động lựa chọn các giải pháp phù hợp với công ty hay ngành của mình để hạn chế rủi ro tài chính nhằm giúp doanh nghiệp phát triển trong bối cảnh nền kinh tế có nhiều thay đổi.

\subsection{Hạn chế và đề nghị}

Bài viết này chỉ tập trung vào các doanh nghiệp ngành bất động sản niêm yết trên HSX trong giai đoạn từ 2012-2018, vì vậy, kết quả nghiên cứu có thể chưa bao quát hết toàn bộ các doanh nghiệp đã niêm yết. Theo tác giả, để khắc phục hạn chế trên, bài viết có thể mở rộng thêm đối tượng nghiên cứu: Hoặc tất cả doanh nghiệp trong ngành bất động sản hoặc tất cả các doanh nghiệp cổ phần niêm yết trên cả sở giao dịch chứng khoán Hà Nội (HNX), sở giao dịch chứng khoán Thành phố Hồ Chí Minh (HSX) và trên UPCOM với thời gian có thể dài hơn.

\section{Tài liệu tham khảo}

Bathory, A. (1984). Predicting corporate collapse: Credit Analysis in the determination and forecasting of insolvent companies. London, UK: Financial Times Business Information.

Bhunia, A., \& Mukhuti, S. (2012). Financial risk measurement of small and medium - Sized companies listed in Bombay stock exchange. International Journal of Advances in Management and Economics, 1(3), 27-34.

Gang, F., \& Dan, D. (2012). Research on the influence factors of financial risk for small and medium - Sized enterprise: An empirical analysis from 216 companies of small plates, ShenZhen stock exchange, China. Journal of Contemporary Research in Business, 3(9), 380-387.

Gang, F., Weilan, F. W., \& Liu, D. (2012). Empirical study on financial risk factors: Capital structure, operation ability, profitability, and solvency - Evidence from listed companies in China. Journal of Business Management and Economics, 3(5), 173-178.

Levin, A., \& Lin, C.-F., \& Chu, C.-S. J. (2002). Unit root tests in panel data: Asymptotic and finite-sample properties. Journal of Econometrics, 108(1), 1-24.

Li, S. (2003). Future trends and challenges of financial risk management in the digital economy. Managerial Finance, 29(5), 111-125.

Napp, A.-K. (2011). Financial management in SME - The use of financial analysis for identifying analysing and monitoring internal financial risks (Master's thesis, Aarhus University, Aarhus, Denmark). Retrieved March 15, 2020, from https://www.coursehero.com/file/8877421/Thesis-A-Napp/ 
Ross, S., Westerfield, R., Jaffe, J., \& Jordan, B. (2016). Corporate finance (11th ed.). New York, NY: McGraw-Hill Education.

Simantinee, S., \& Phani, T. V. V. K. (2015). Factors influencing financial risk - A case of NSE NIFTY Companies. International Journal in Management and Social Science, 3(8), 132-137.

Vu, H. T. (2017). Phân tích các yếu tố ảnh hưởng đến rủi ro tài chính của các công ty niêm yết ngành bất động sản tại Sở giao dịch chứng khoán Thành phố Hồ Chí Minh [Analysis of factors affecting financial risks of real estate listed companies on the Ho Chi Minh City Stock Exchange]. Tạp chí Kinh tế \& Phát triển, 240, 86-93.

Zhe, L., Ke, L., Kaibi, W., \& Xiaoliu, S. (2012). Research on financial risk management for electric power enterprises. Systems Engineering Procedia, 4, 54-60. 